\title{
RESEARCH
}

Open Access

\section{Characterizing cardiac involvement in amyloidosis using cardiovascular magnetic resonance diffusion tensor imaging}

\author{
Alexander Gotschy ${ }^{1,2+}$, Constantin von Deuster ${ }^{1 \dagger}$, Robbert J. H. van Gorkum¹, Mareike Gastl ${ }^{1}$, Ella Vintschger ${ }^{1}$,
} Rahel Schwotzer ${ }^{3}$, Andreas J. Flammer ${ }^{2}$, Robert Manka ${ }^{2,4}$, Christian T. Stoeck ${ }^{1}$ and Sebastian Kozerke ${ }^{1 *}$

\begin{abstract}
Background: In-vivo cardiovascular magnetic resonance (CMR) diffusion tensor imaging (DTI) allows imaging of alterations of cardiac fiber architecture in diseased hearts. Cardiac amyloidosis (CA) causes myocardial infiltration of misfolded proteins with unknown consequences for myocardial microstructure. This study applied CMR DTI in CA to assess microstructural alterations and their consequences for myocardial function compared to healthy controls.

Methods: Ten patients with CA (8 AL, 2 ATTR) and ten healthy controls were studied using a diffusion-weighed second-order motion-compensated spin-echo sequence at 1.5 T. Additionally, left ventricular morphology, ejection fraction, strain and native T1 values were obtained in all subjects. In CA patients, T1 mapping was repeated after the administration of gadolinium for extracellular volume fraction (ECV) calculation. CMR DTI analysis was performed to yield the scalar diffusion metrics mean diffusivity (MD) and fractional anisotropy (FA) as well as the characteristics of myofiber orientation including helix, transverse and E2A sheet angle (HA, TA, E2A).

Results: MD and FA were found to be significantly different between CA patients and healthy controls (MD $1.77 \pm$ $0.1710^{-3}$ vs $1.41 \pm 0.0710^{-3} \mathrm{~mm}^{2} / \mathrm{s}, p<0.001$; FA $0.25 \pm 0.04$ vs $0.35 \pm 0.03, p<0.001$ ). MD demonstrated an excellent correlation with native $\mathrm{T} 1(r=0.908, p<0.001)$ while FA showed a significant correlation with ECV in the CA population ( $r=-0.851, p<0.002$ ). HA exhibited a more circumferential orientation of myofibers in CA patients, in conjunction with a higher TA standard deviation and a higher absolute E2A sheet angle. The transmural HA slope was found to be strongly correlated with the global longitudinal strain $(r=0.921, p<0.001)$.

Conclusion: CMR DTI reveals significant alterations of scalar diffusion metrics in CA patients versus healthy controls. Elevated MD and lower FA values indicate myocardial disarray with higher diffusion in CA that correlates well with native T1 and ECV measures. In CA patients, CMR DTI showed pronounced circumferential orientation of the myofibers, which may provide the rationale for the reduction of global longitudinal strain that occurs in amyloidosis patients. Accordingly, CMR DTI captures specific features of amyloid infiltration, which provides a deeper understanding of the microstructural consequences of CA.
\end{abstract}

Keywords: Cardiovascular magnetic resonance imaging, Diffusion tensor imaging, Cardiac amyloidosis, Myocardial microstructure, Tissue characterization

\footnotetext{
* Correspondence: kozerke@biomed.ee.ethz.ch

${ }^{\dagger}$ Alexander Gotschy and Constantin von Deuster contributed equally to this

work.

${ }^{1}$ Institute for Biomedical Engineering, University and ETH Zurich, Gloriastrasse

35, Zurich 8092, Switzerland

Full list of author information is available at the end of the article
}

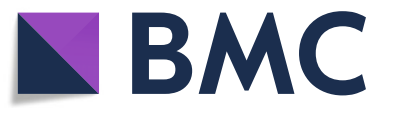

(c) The Author(s). 2019 Open Access This article is distributed under the terms of the Creative Commons Attribution 4.0 International License (http://creativecommons.org/licenses/by/4.0/), which permits unrestricted use, distribution, and

reproduction in any medium, provided you give appropriate credit to the original author(s) and the source, provide a link to the Creative Commons license, and indicate if changes were made. The Creative Commons Public Domain Dedication waiver (http://creativecommons.org/publicdomain/zero/1.0/) applies to the data made available in this article, unless otherwise stated. 


\section{Introduction}

Amyloidosis is a multi-system disease that is caused by the synthesis and accumulation of unstable and misfolded proteins leading to a loss of normal tissue architecture and function [1]. While almost every organ can be affected by amyloid deposition, the prognosis of patients is mainly determined by the occurrence and extent of myocardial involvement [2-4]. There are various types of amyloid that differ in the source and nature of the misfolded precursor protein. Of those, light chain associated amyloidosis (AL) and transthyretin amyloid amyloidosis (ATTR) are the two main types of amyloidosis that affect the heart. The infiltration of amyloid to the heart leads to myocardial thickening and diastolic dysfunction resulting in heart failure.

For the assessment of cardiac amyloidosis (CA), cardiac imaging plays a fundamental role [5]. Besides myocardial thickening, echocardiography often shows a reduction of global longitudinal strain, which typically spares the apical segments [6]. Non-invasive characterization of the myocardial tissue of amyloidosis patients is the strength of cardiovascular magnetic resonance (CMR). In particular, the extent and distribution of late gadolinium enhancement (LGE) has been shown to provide independent information on outcome even after adjustment for known prognostic factors [7]. CA is also an ideal application for newer mapping techniques as it leads to excessively high T1- and extracellular volume (ECV) $[8,9]$. Notably, in AL CA patients, ECV is a proven predictor of mortality with an up to four-fold increased likelihood of death for ECV $>0.45$ over a median follow-up period of 23 months [10]. Both LGE and ECV, however, rely on the application of gadolinium-based contrast agents (GBCA), which is often limited in amyloidosis patients due to severely reduced renal function.

Recently, CMR diffusion tensor imaging (DTI) emerged as a promising method for the determination of myocardial fiber orientation [11-13] and tissue characterization without the need for GBCA. It allows to investigate water diffusion within the tissue and to derive additional scalar metrics, such as mean diffusivity (MD) and fractional anisotropy (FA) for the quantification of structural integrity $[14,15]$. Alterations of cardiac microstructure have been described in patients with dilated cardiomyopathy (DCM), myocardial infarction and hypertrophic cardiomyopathy (HCM) [16-18]. In CA, however, the effect of the double harm resulting from the combination of amyloid infiltration with direct cardio-toxicity of abnormal proteins [19] on the myocardial structure and its potential impact on diffusion properties is yet unknown.

The objective of this study was to determine the consequences of amyloid infiltration on myocardial microstructure in-vivo using CMR DTI and to investigate their effect on myocardial function. Scalar diffusion and relaxation metrics (MD/FA and native T1/ECV, respectively) were correlated in CA patients and controls. In addition, directional diffusion information was assessed to reveal changes in myofiber orientations and compared to parameters of left ventricular (LV) function.

\section{Methods \\ Study design}

Between March 2017 and June 2018, 12 patients with AL or ATTR CA ( $63 \pm 11$ years, 3 female), referred to the local outpatient clinic, and 10 healthy, age-matched controls $(62 \pm 11$ years, 4 female) were prospectively enrolled. Exclusion criteria were cardiovascular disease other than CA, kidney failure with estimated glomerular filtration rate $(\mathrm{eGFR})<30 \mathrm{ml} / \mathrm{min} / 1.76 \mathrm{~m}^{2}$ and the standard exclusion criteria for CMR [20]. Height and weight of all study subjects were recorded to calculate the body surface area (BSA). All patients had histologically proven (positive Congo red staining of endomyocardium, abdominal fat, kidney, rectum or bone marrow biopsies) amyloidosis and echocardiographic or CMR findings typical for cardiac involvement. Imaging was performed on a clinical 1.5 $\mathrm{T}$ system (Achieva, Philips Healthcare, Best, The Netherlands) equipped with a 5-channel cardiac receiver array. Prior to imaging, written informed consent was obtained from all subjects. The study protocol was approved by the ethics committee of the canton of Zurich; it allowed the administration of GBCA only in the CA patient group.

\section{CMR data acquisition}

Cardiac function was assessed by a contiguous stack of balanced steady-state free precession (bSSFP) short-axis cine images covering the entire LV and bSSFP long-axis cine images in 2-chamber, 3-chamber and 4-chamber orientations. According to the cine images, systolic quiescent time points were determined on a per subject basis.

Diffusion-weighted imaging was performed with a single-shot spin-echo sequence utilizing second-order motion compensated diffusion sensitizing gradients in combination with an echo planar imaging (EPI) readout $[21,22]$. The sequence was electrocardiogram (ECG)triggered to mid-systole systole (65\% of peak systole) and three imaging planes were placed in short-axis view orientation at apical, mid-ventricular and basal level LV using a reduced field-of-view (FOV) technique [23]. Diffusion weighting was encoded along nine and three directions with a b-value of 450 and $100 \mathrm{~s} / \mathrm{mm}^{2}$, respectively [24]. Data acquisition was performed during free breathing with respiratory navigator-based slice tracking [25]. The imaging parameters are listed in Table 1.

T1 mapping was performed using a modified LookLocker inversion (MOLLI) sequence with a total of eight 
Table 1 CMR Scan Parameters

\begin{tabular}{llll}
\hline & DTI & T1 Mapping & LGE \\
\hline Spatial Resolution $\left[\mathrm{mm}^{3}\right]$ & $2.5 \times 2.5 \times 8$ & $1.2 \times 1.2 \times 8$ & $1.6 \times 1.6 \times 10$ \\
FOV $\left[\mathrm{mm}^{2}\right]$ & $230 \times 105$ & $300 \times 300$ & $360 \times 460$ \\
TR & 3 beats & $2.5 \mathrm{~ms}$ & $3.5 \mathrm{~ms}$ \\
TE $[\mathrm{ms}]$ & 76 & 1 & 1.7 \\
Flip Angle $\left[^{\circ}\right]$ & 90 & 35 & 15 \\
Number of Averages & 12 & 1 & 1 \\
Respiratory Mode & Free Breathing & Breath Hold & Breath Hold \\
Approx. Scan Time [min.] & $8-10$ min & $1-2$ min & 1 min \\
\hline
\end{tabular}

DTI diffusion tensor imaging, FOV field of view, LGE late gadolinium enhancement, $T E$ echo time, $T R$ repetition time

T1 weighted images split into a set of five and three images acquired after inversion, separated by three heartbeats of recovery (5-(3)-3 scheme) [26]. The imaging planes were at the same slice location as the DTI scan. In all subjects, native T1 maps were acquired. For ECV calculation, T1 mapping was repeated in the patient group $20 \mathrm{~min}$ after a bolus injection of $0.2 \mathrm{mmol} / \mathrm{kg}$ gadolinium-based contrast agent (Gadovist, Bayer Schering, Berlin, Germany). The hematocrit was measured in all patients within $2 \mathrm{~h}$ of CMR. LGE images were acquired with an inversion-recovery sequence approximately $10 \mathrm{~min}$ after the bolus injection.

\section{CMR data analysis}

Standard measurements of LV size and function including $\mathrm{LV}$ ejection fraction (LVEF), left ventricular mass index (LVMi) and intraventricular septal wall thickness (IVS) were obtained using the GTVolume software package (GyroTools LLC, Zurich, Switzerland). The LV endsystolic global longitudinal strain (GLS) and global circumferential strain (GCS) were calculated using a certified CMR feature tracking evaluation software (2 D CPA MR, Cardiac Performance Analysis MR Version 4, TomTec Imaging Systems, Unterschleissheim, Germany) on the long- and short-axis bSSFP images.

Prior to diffusion tensor calculation and T1 fitting, the individual MOLLI and DTI images were registered using a non-rigid groupwise image registration method [27] for compensation of residual motion. DTI analysis was performed on MD, FA, helix angle (HA), transverse angle (TA) and absolute E2A sheet angle $[16,28]$. A detailed description of the DTI parameters is provided as Additional file 1. For calculating the HA slope, a linear regression of the transmural course of the helix angles between the endocardium and epicardium was performed, excluding boundary pixels at the endo- and epicardium. Angular diffusion metrics were evaluated in four radial and circumferential sectors of the left ventricle and averaged across all three slices. Mean LV signal-to- noise ratio (SNR) of the $b=100 \mathrm{~s} / \mathrm{mm}^{2}$ image was determined in both patient groups for quality control. The scalar diffusion metrics $\mathrm{MD}$ and FA as well as native $\mathrm{T} 1$ and ECV, were evaluated in a single septal region-of-interest (ROI) on the midventricular shortaxis slices in accordance with current clinical recommendations [29]. ECV was computed based on the collected haematocrit, native and enhanced T1 values in the myocardium and the blood pool.

\section{Statistical analysis}

All data are expressed as mean \pm SD. Statistical analysis was conducted using MedCalc software (MedCalc 17.9.7, MedCalc Software bvba, Ostend, Belgium). Differences in diffusion and relaxation metrics between patients and controls were assessed by unpaired two-tailed t-tests. To determine statistical associations between parameters, Pearson correlation analyses were performed. In addition, the diagnostic accuracy of native T1, MD and FA for detecting cardiac amyloidosis compared to healthy controls was examined by ROC analysis. A p-value less than 0.05 was considered statistically significant.

\section{Results}

\section{Patient characteristics}

Data from two of 12 CA subjects were excluded, one for difficulty with breath-holding and one for technical reasons. Ten CA patients ( $60 \pm 11$ years, 7 male) were hence included in the analysis. Eight patients suffered from ALamyloidosis while two had ATTR-amyloidosis (one wild type, one heterozygous mutation c.238A > G). The diagnosis of CA was confirmed by endomyocardial biopsy $(n=4)$ or the combination of typical imaging findings (echocardiography or CMR) with proven amyloid deposition in bone marrow $(n=3)$, kidney $(n=1)$, rectum $(n=1)$ or abdominal fat $(n=1)$ biopsies. The renal function was normal in two patients, while there were five patients with mildly and three patients with moderately reduced renal function (eGFR: $70 \pm 21 \mathrm{ml} / \mathrm{min}$ ). The age- and sex-matched control group also showed similar BSA characteristics. The IVS $(14.4 \pm 2.6$ vs $8.0 \pm 1.3 \mathrm{~mm}, p<0.001)$ and the LVMi $\left(79 \pm 19\right.$ vs $\left.44 \pm 7 \mathrm{~g} / \mathrm{m}^{2}, p<0.001\right)$ were significantly elevated in CA patients. While GLS was significantly impaired in CA patients $(-16.6 \pm 3.3$ vs $-22.4 \pm 1.9 \%, p<$ $0.001), \operatorname{GCS}(-26.9 \pm 5.5$ vs $-29.1 \pm 3.1 \%, p=0.30)$ and LVEF ( $64 \pm 6$ vs $61 \pm 4 \%, p=0.20$ ) exhibited no significant difference between the groups. Table 2 contains the baseline characteristics of both groups.

Comparison of scalar DTI parameters with ECV and native T1 Midventricular septal reference values, obtained in the healthy controls were $1.41 \pm 0.0710^{-3} \mathrm{~mm}^{2} / \mathrm{s}$ for $\mathrm{MD}$, $0.35 \pm 0.03$ for FA and $1020 \pm 42 \mathrm{~ms}$ for native T1. In the 
Table 2 Baseline characteristics of the Study Population

\begin{tabular}{llll}
\hline & $\begin{array}{l}\text { CA } \\
\text { Patients } \\
N=10\end{array}$ & $\begin{array}{l}\text { Healthy } \\
\text { Controls } \\
N=10\end{array}$ & $p$-value \\
& $60 \pm 11$ & $62 \pm 11$ & 0.79 \\
\hline Age [years] & $7(70)$ & $6(60)$ & 0.73 \\
Male (\%) & $1.80 \pm 0.13$ & $1.85 \pm 0.18$ & 0.50 \\
BSA [m²] & $8(80)$ & & \\
AL-Amyloidosis & $2(20)$ & & \\
ATTR-Amyloidosis & $14.4 \pm 2.6$ & $8.0 \pm 1.3$ & $<0.001$ \\
IVS [mm] & $134 \pm 25$ & $133 \pm 20$ & 0.97 \\
LVEDV [ml] & $79 \pm 19$ & $44 \pm 7$ & $<0.001$ \\
LVMi [g/m2] & $64.0 \pm 6.3$ & $60.7 \pm 3.9$ & 0.20 \\
LVEF [\%] & $-16.6 \pm 3.3$ & $-22.4 \pm 1.9$ & $<0.001$ \\
GLS [\%] & $-26.9 \pm 5.5$ & $-29.1 \pm 3.1$ & 0.30 \\
GCS [\%] & & &
\end{tabular}

Data are mean \pm SD or number of patients (\%)

AL amyloid light chain, ATTR transthyretin-associated amyloidosis, BSA body surface area, CA cardiac amyloidosis, GCS global circumferential strain, GLS global longitudinal strain, IVS interventricular septum thickness in diastole, LVEDV left ventricular end-diastolic volume, LVEF left ventricular ejection fraction, LVMi left ventricular mass indexed to body surface area

CA group, a significantly higher native T1 $(1136 \pm 61 \mathrm{~ms}$, $p<0.001)$, highly elevated MD $\left(1.77 \pm 0.1710^{-3} \mathrm{~mm}^{2} / \mathrm{s}\right.$, $p<0.001)$ and significantly reduced FA $(0.25 \pm 0.04, p<$ 0.001 ) were found when comparing with the healthy control group. ECV in the CA group was elevated to
$0.50 \pm 0.14$. Figure 1 depicts representative mid-ventricular slices of MD, FA and native T1 of a healthy control and an AL-amyloidosis case with additional ECV and LGE images in the CA patient. Comparing the different modalities within the CA patient, it can be appreciated that the areas of LGE hyperenhancement and elevated ECV correspond well with the regions of high MD and low FA in the parameter maps derived from DTI.

Direct comparison between native T1 values versus MD over the whole study population demonstrates an excellent correlation between the two parameters ( $r=$ 0.909, 95\% CI [0.778, 0.963], $p<0.001$, Fig. 2a). A weaker, but still significant negative correlation was also found between native T1 and FA ( $r=-0.715$, 95\% CI [$0.879,-0.398$ ]; $p<0.001$; Fig. $2 \mathrm{~b}$ ). The analysis of correlation between DTI-based parameters and ECV was limited to the CA group. It exhibited a fair correlation between ECV and MD ( $r=0.744,95 \%$ CI [0.216, 0.936]; $p=0.014$; Fig. 2c) and a strong negative correlation between ECV and FA $(r=-0.851,95 \%$ CI [ $-0.964,-0.477]$; $p<0.002$; Fig. 2d).

For the differentiation between healthy controls and CA patients, the scalar DTI parameters exhibit promising accuracy with an area under the curve (AUC) of 0.96 [95\% CI, 0.77-1.00] for MD and 0.97 [95\% CI, 0.78-1.00] for FA which were not significantly different from the diagnostic performance of native T1 (AUC 0.91 [95\% CI,

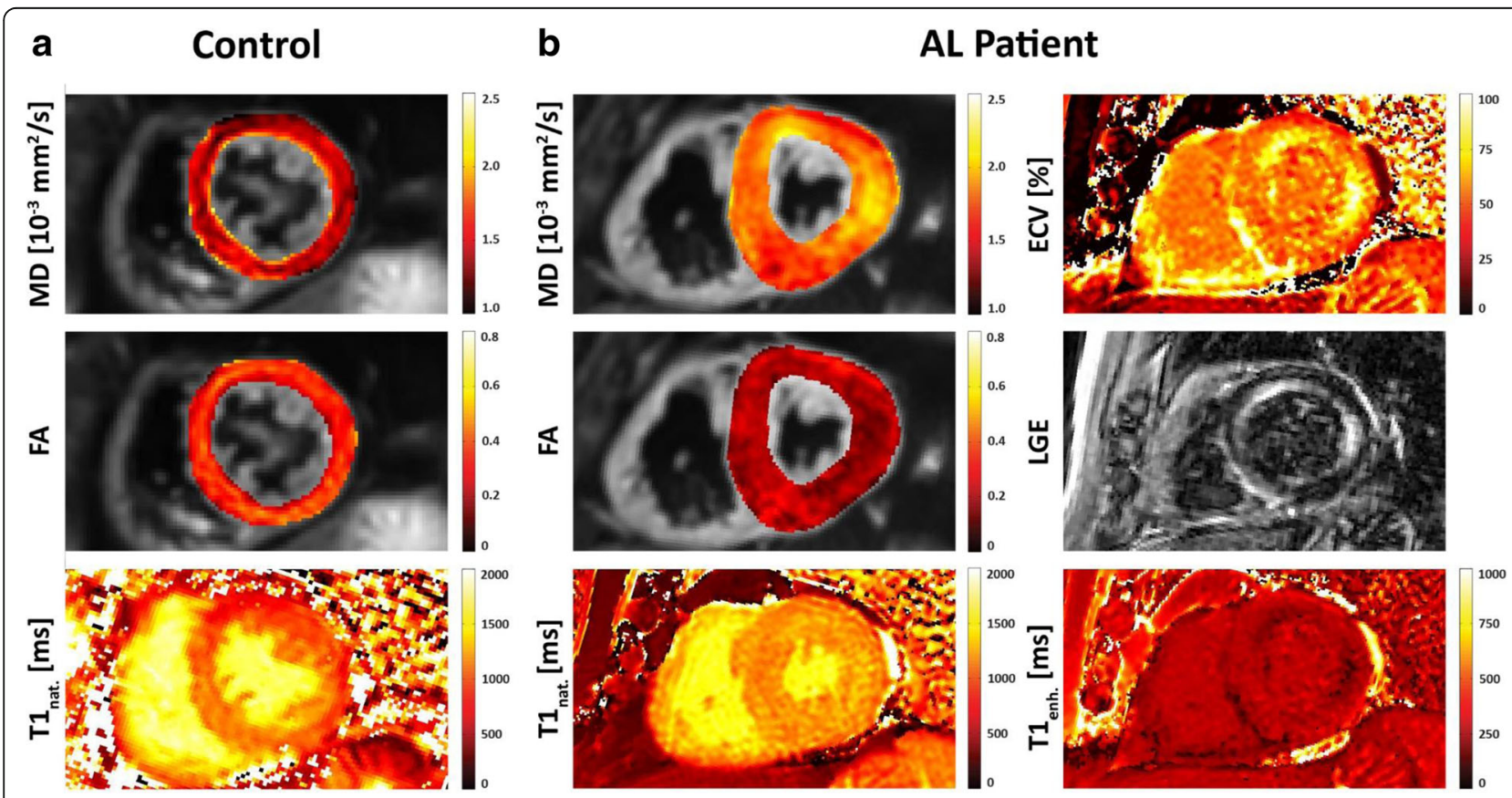

Fig. 1 Representative mean diffusivity (MD), fractional anisotropy (FA) and native T1 maps for a healthy control (a) and a light chain (AL) amyloidosis patient (b). Enhanced T1 mapping, extracellular volume fraction (ECV) mapping and late gadolinium enhancement (LGE) CMR imaging was only performed in the cardiac amyloid (CA) patient. It can be appreciated, that the areas of signal hyperenhancement in LGE and elevated ECV correspond well with regions of high MD and low FA in the parameter maps derived from CMR diffusion tensor imaging (DTI) in the CA patient 

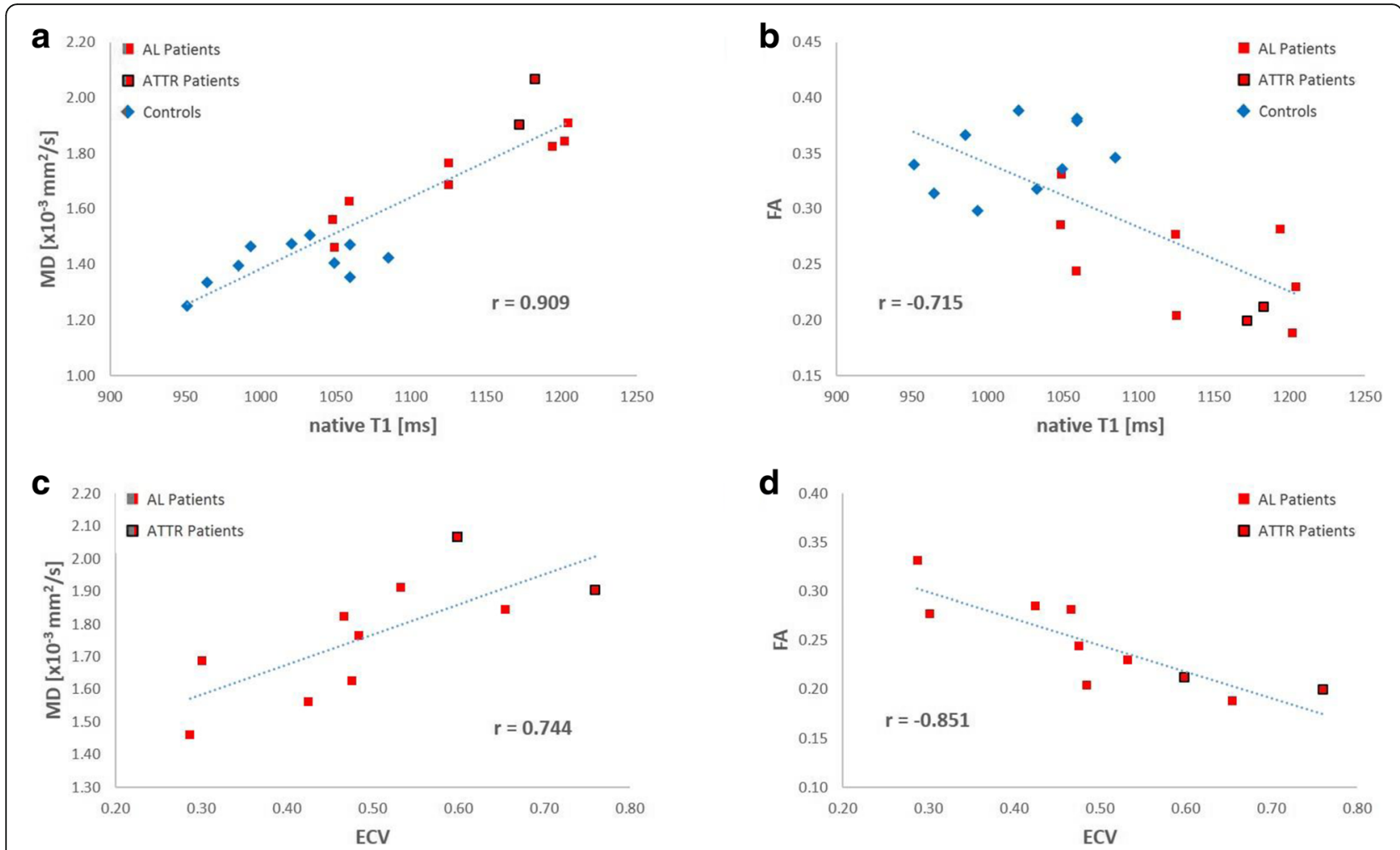

Fig. 2 Correlation plots for MD and FA with native T1 and ECV. Native T1 shows an excellent correlation with MD (a) and a moderate but still significant negative correlation with FA (b). Within the patient population, MD is also correlated with ECV (c) but FA exhibits a higher correlation with ECV $(\mathbf{d})$

0.69-0.99]). Additional file 2: Figure S2 provides detailed information on the AUC analysis (Additional file 2).

\section{Evaluation of myocardial microstructure in cardiac amyloidosis}

Figure $3 \mathrm{a}$ depicts the comparison of the HA, TA and absolute E2A sheet angle of a representative CA patient and a healthy control. At first sight, despite the thicker myocardium in CA patients, the distribution of $\mathrm{HA}$ seems similar between both groups (Fig. 3a first row). Quantitative evaluation, however, reveals that the transmural HA slope is significantly reduced in CA patients $(-0.83 \pm 0.16 \%$ transmural depth (tmd) vs $-1.02 \pm$ $\left.0.14 \% \%_{\text {tmd }} ; p<0.001\right)$. In case of the patients, this leads to a reduced longitudinal orientation of fibers in the epicardial and endocardial layers compared to the controls as shown in the bullseye plots in Fig. 3b first row.

The TA is close to zero degrees in both groups with no significant difference between CA patients and healthy controls $\left(4.5 \pm 4.6\right.$ vs $\left.2.5 \pm 3.6^{\circ}, p=0.07\right)$. The standard deviation per segment, however, is significantly larger in the CA patients $\left(18.2 \pm 6.6\right.$ vs $\left.12.7 \pm 2.6^{\circ}, p<0.001\right)$ indicating a higher level of deviation from the circumferential alignment of the myocardial fibers found in healthy subject myocardium. Representative TA maps are shown in Fig. 3a middle row. The higher TA standard deviation can be appreciated by the more irregular appearance of the TA map in the CA patient and the quantitative overview in Fig. 3b middle row.

The most prominent difference in the myocardial microstructure between CA patients and healthy controls becomes evident in the comparison of the absolute E2A sheet angle. Patients exhibited higher mean absolute E2A sheet angles in every segment with values approximately $20^{\circ}$ greater than those of healthy subjects $(52.9 \pm 7.5$ vs $33.9 \pm 9.5^{\circ}, p<0.001$, Fig. 3ab lower row). Average SNR of a single $\mathrm{b}=100 \mathrm{~s} / \mathrm{mm}^{2}$ image was comparable between patients and controls $(12.0 \pm 3.0$ vs $12.5 \pm 3.6, p=0.62)$.

\section{Correlation between myocardial microstructure and left ventricular function}

HA slope and E2A sheet angle were correlated against LVEF, GLS and GCS. A strong correlation was found between HA slope and GLS $(r=0.921,95 \%$ CI [0.809, 0.969]; $p<0.0001$; Fig. 4a), indicating impaired longitudinal function with a flatter transmural HA gradient. A weaker, but still significant correlation could be observed between E2A sheet angle and GLS ( $r=0.763$, 95\% CI [0.483, 0.901]; $p=0.0001 ;$ Fig. 4b). LVEF and GCS 


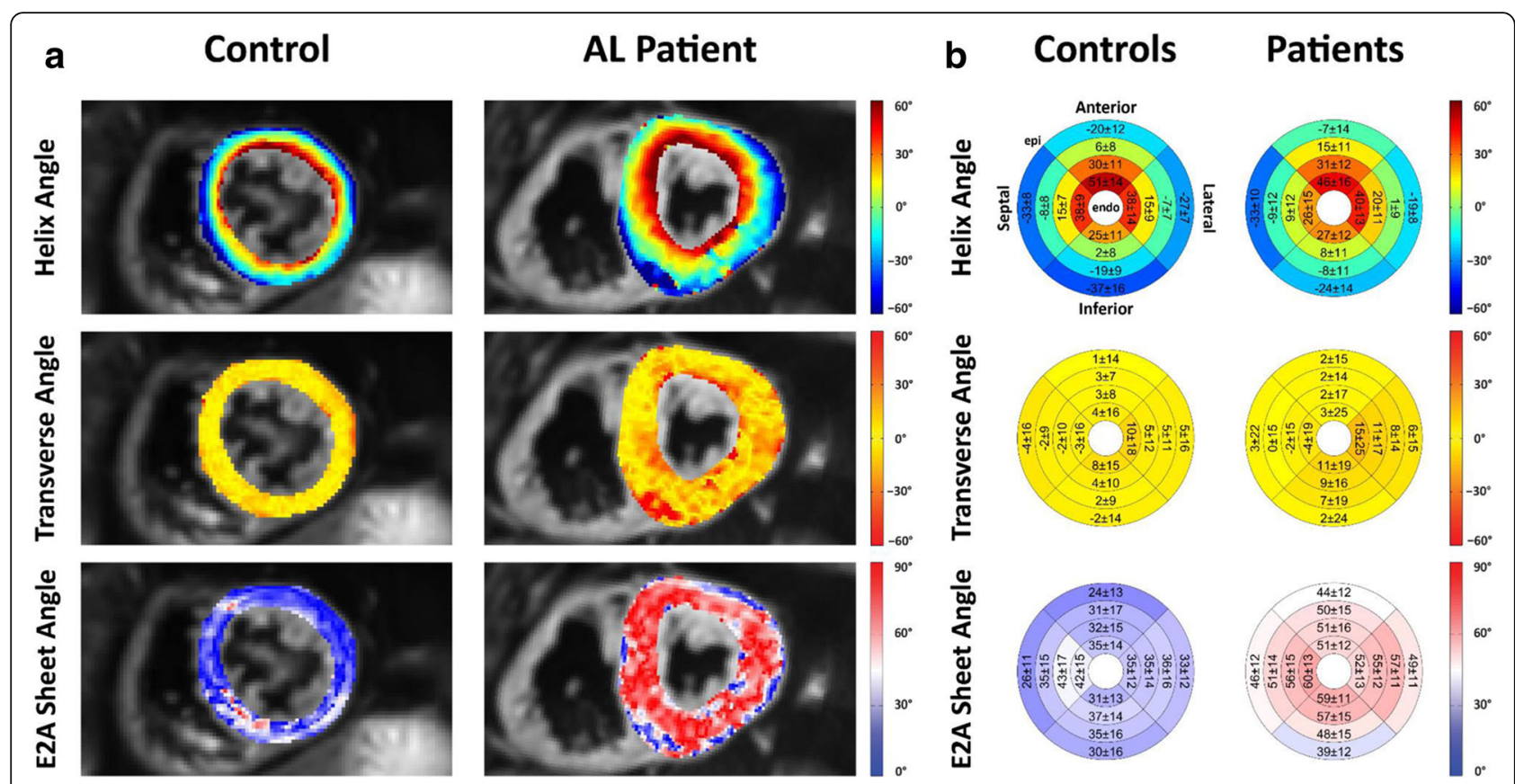

Fig. 3 Example helix angle (HA), transverse angle (TA) and sheet angle (E2A) map for a healthy control subject and an AL-amyloidosis patient (a). Image (b) shows Bullseye plots for HA, TA and E2A across all subjects for each cohort. Reported values are mean \pm SD

exhibited no significant correlation with any parameter describing the myocardial microstructure.

\section{Discussion}

This study assessed the value of cardiac DTI for the evaluation of patients with CA and investigated the effects of amyloid deposition on myocardial microstructure.

The scalar DTI metrics of MD and FA were found to be significantly altered by CA. In comparison with native $\mathrm{T} 1$, which was also elevated in CA patients in agreement with previous studies [10,30], MD and FA exhibited a comparable AUC to identify CA patients indicating potential for similar diagnostic accuracy. The correlation between $\mathrm{MD}$ and native T1 suggests that the pathomechanism of amyloid infiltration affects both parameters to a similar extent. While native $\mathrm{T} 1$ is a useful diagnostic marker for CA, recent studies have shown that it is inferior to ECV as an independent predictor of prognosis in AL-amyloidosis as well as ATTR-amyloidosis patients $[30,31]$. However, the significant number of amyloidosis patients suffering from renal failure stresses the need for a gadolinium-free substitute for ECV with potential prognostic value. The robust correlation between FA and ECV found in the CA patients justifies expectations, that FA may have potential to serve as such a substitute for ECV. This warrants future trials to investigate the diagnostic accuracy of FA and MD for amyloidosis in particular against other cardiomyopathies with a HCM phenotype. The existence of a tight functional relation between FA and ECV is also suggestive from a
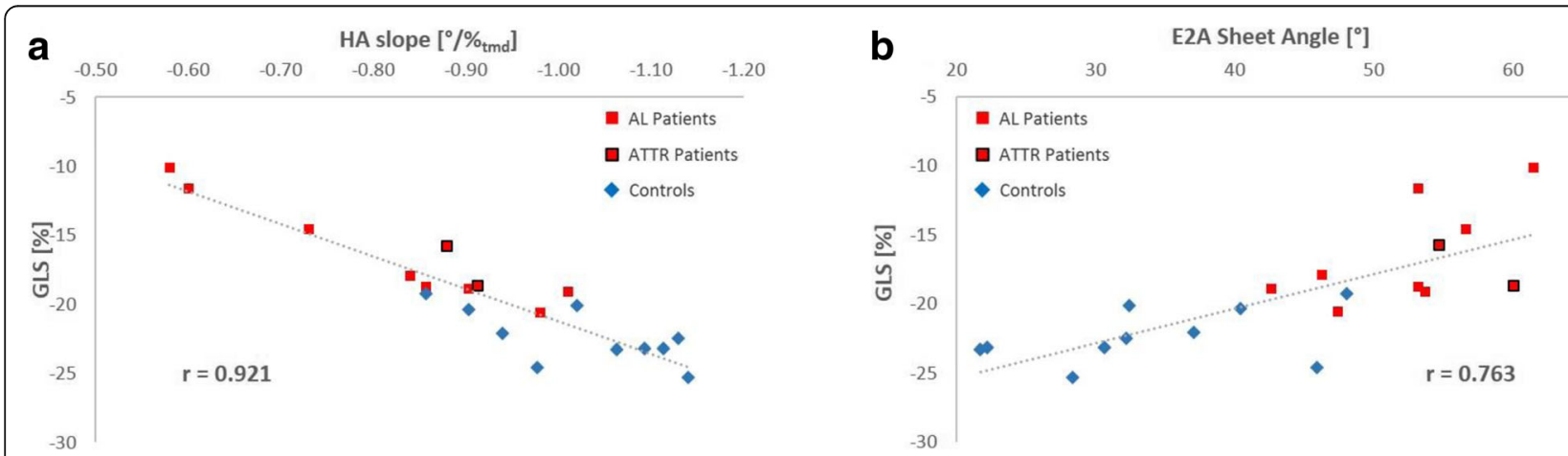

Fig. 4 Correlation plots for GLS with HA slope and E2A sheet angle. The HA slope shows an excellent correlation with GLS (a) while a moderate but still significant correlation between GLS and E2A could be observed (b) 
microstructural perspective, since the scalar CMR DTI parameters describe the range and anisotropy of myocardial water movement, which is mainly determined by the tissue-specific presence of spatially ordered cellular compartments within the myocardium [32]. Our findings are supported by previous studies, which have demonstrated that quantitative measures of diffusivity derived from CMR DTI capture similar properties as LGE and ECV in myocardial infarction and HCM [33, 34].

Time efficiency is an essential criterion for clinical practice under the economic constraints of healthcare systems. From this point of view, measurement durations of approximately ten minutes seem unreasonably long and insufficient for daily routine in CMR imaging. However, the presented sequence acquired three slices, two of which, namely the basal and apical slice were only used for the determination of myocardial microarchitecture. The MD and FA values were only assessed in the mid-ventricular slice as recommended in the current consensus document [29]. Limiting the acquisition to a single mid-ventricular slice would reduce the measurement time by approximately $25 \%$ assuming a $1.5 \mathrm{~T}$ CMR system and a heart rate of 60 beats per minute. In addition, replacing LGE and ECV with DTI would also eliminate the 10-15 min waiting period before LGE and ECV $[35,36]$ imaging and could ultimately lead to a saving of time.

Our investigation of myocardial architecture provided new insights into the microstructural alterations due to interstitial amyloid deposition. The most prominent observation of significantly increased absolute E2A sheet angle is the structural equivalent of a more systolic conformation of the myocardial sheetlets. The correlation between E2A sheet angle and GLS may indicate that, at a microstructural level, a pronounced systolic sheetlet configuration might be associated with a limited ability to rotate to a relaxed conformation in diastole. Similar observations have been made in HCM patients [17], indicating that despite fundamentally different pathogenesis, comparable structural alterations may cause the primary diastolic heart failure observed in both diseases. The difference in HA between health and disease, however, has not yet been described. The significantly lower HA slope of CA patients results in a reduced transmural HA gradient with a less longitudinal orientation of the myocardial fibers. The strong correlation which we observed between the HA slope and the GLS indicates, that the more circumferential myofiber orientation may be the microstructural determinant for the loss of longitudinal function in amyloidosis patients. This finding seems not to be a common feature of all hypertrophic phenotype cardiomyopathies as Nielles-Vallespin et al. described similar HA configuration in HCM patients and healthy controls [17]. In agreement with the hypothesis of the HA slope being a determinant for longitudinal function, a recent study by Pagourelias et al. demonstrated that the GLS of CA patients is significantly lower compared to HCM patients with identical myocardial wall thickness [37]. In addition to the microstructural alterations, the direct cardio-toxicity of amyloid deposition may also contribute to a loss of myocardial function in CA patients [19].

It remains unclear whether the relation between HA slope and GLS is linear over a wider range. A previous study by von Deuster et al. [16] in DCM patients reported similar HA slope in controls and patients with mildly reduced LVEF, but two patients with advanced heart failure showed reduced GLS and increased HA slope. In synopsis, these findings support the hypothesis of a U-shaped relation between HA slope and GLS with an ideal HA slope around $1 \% \%_{\text {tmd }}$ and impaired longitudinal function caused by deviations to significantly higher and lower HA slope values. The different constellations of DTI parameters observed in HCM, DCM and amyloidosis raise the hope that future research may identify disease specific patterns of DTI parameters, which would be an advantage over rather unspecific parameters such as native T1 that is elevated in a multitude of cardiac diseases. The presence of subtype specific patterns of the DTI parameters for AL- and ATTR-amyloidosis should be investigated, in particular in comparison to nuclear imaging techniques where radiotracers used for technetium-labeled bone scintigraphy are found to have high sensitivity for ATTR [38], while ${ }^{18} \mathrm{~F}$ florbetaben-PET has recently been shown to have a high sensitivity for AL-amyloidosis [39].

In contrast to $\mathrm{HA}$ and $\mathrm{E} 2 \mathrm{~A}$, the transverse angle does not seem to provide relevant insights into disease specific pathologies as it is distributed around $0^{\circ}$ in health and disease. However, the elevated standard deviation of TA that we observed in amyloidosis patients has previously already been observed in DCM patients [16] and may therefore be a surrogate for myocyte disarray of any cause. A bias of the TA distribution due to low SNR, which has been observed in previous studies [24], could be excluded in our work since SNR values were not different between the amyloidosis and control cohorts.

\section{Limitations}

A limitation of the present work is the small sample size of CA patients with different types of the disease. In particular the patient number is insufficient to investigate potential effects of the different pathophysiologies of AL- and ATTR-amyloidosis on the myocardial microstructure. In addition, the lack of GBCA application in healthy controls, due to constraints from ethical approval constitutes a limitation. However, despite those limitations, our patient population already showed promising diagnostic properties of CMR DTI based 
parameters warranting future research in a larger population of CA patients. In contrast to previous studies using STEAM based DTI methods [40], this study was limited to investigate the mid-systolic configuration of the myocardium and did not assess a diastolic state.

\section{Conclusion}

The scalar CMR DTI parameters MD and FA in CA patients are significantly different from healthy controls, allowing to assess the amyloid induced microstructural disarray without the use of GBCA. The major differences in myofiber orientation are a significantly increased E2A sheet angle and a reduced HA slope in CA patients. Finally, the strong correlation between HA slope and GLS indicates, that the more circumferential orientation of the myofibers may provide the rationale for the reduction of CLS that occurs in CA.

\section{Additional files}

Additional file 1: Figure S1. (PDF $482 \mathrm{~kb}$ )

Additional file 2: Figure S2. (PDF $61 \mathrm{~kb}$ )

\begin{abstract}
Abbreviations
AL: Amyloid light chain; ATTR: Transthyretin-associated amyloidosis; AUC: Area under the curve; BSA: Body surface area; bSSFP: balanced steady-state free precession; CA: Cardiac amyloidosis; CMR: Cardiovascular magnetic resonance; DCM: Dilated cardiomyopathy; DTI: Diffusion tensor imaging; E2A: E2A sheet angle; ECG: Electrocardiogram; ECV: Extracellular volume; EDV: End-diastolic volume; EGFR: Estimated glomerular filtration rate; EPI: Echo planar imaging; FA: Fractional anisotropy; FOV: Field of view; GBCA: Gadolinium based contrast agent; GCS: Global circumferential strain; GLS: Global longitudinal strain; HA: Helix angle; HCM: Hypertrophic cardiomyopathy; IVS : Interventricular septum thickness; LGE: Late gadolinium enhancement; LV: Left ventricle/left ventricular; LVEF: Left ventricular ejection fraction; LVMi: Left ventricular indexed mass; MD: Mean diffusivity; MOLLI: Modified Look Locker inversion recovery; ROC: Receiver operating characteristic; RV: Right ventricle/right ventricular; RVEF: Right ventricular ejection fraction; SD: Standard deviation; SV: Stroke volume; TA: Transverse angle; TE: Echo time; TR: Repetition time
\end{abstract}

\section{Acknowledgements}

Not applicable

\section{Authors' contributions}

AG, CVD, RM; CTS and SK conceived the study design. AG, CVD, MG, and CTS performed data acquisition. CVD, RVG and CTS developed CMR sequences, image reconstruction and post-processing. AG, CVD, EV and CTS analyzed CMR scans and the data. AG and CVD drafted the manuscript. AG, CVD, RVG, $M G, E V, R S, A F, R M, C T S$ and SK critically revised the manuscript. All authors read and approved the final manuscript.

\section{Funding}

This work was supported by grants from the Swiss National Science Foundation SNF CR2313_166485 and PZOOP2_174144.

\section{Availability of data and materials}

The datasets used and analyzed during the current study are available from the corresponding author on reasonable request.

\section{Ethics approval and consent to participate}

The study design was approved by the local ethics committee (Kantonale Ethikkommission Zürich; No. EK 2013-0132) and was conducted according to the Declaration of Helsinki. Written informed consent was obtained from all study participants.

\section{Consent for publication}

Not applicable

\section{Competing interests}

The authors declare that they have no competing interests.

\section{Author details}

${ }^{1}$ Institute for Biomedical Engineering, University and ETH Zurich, Gloriastrasse 35, Zurich 8092, Switzerland. ${ }^{2}$ Department of Cardiology, University Hospital Zurich, Zurich, Switzerland. ${ }^{3}$ Division of Medical Oncology and Hematology, University Hospital Zurich, Zurich, Switzerland. Institute of Diagnostic and Interventional Radiology, University Hospital Zurich, Zurich, Switzerland.

Received: 14 February 2019 Accepted: 15 July 2019

Published online: 05 September 2019

\section{References}

1. Eisenberg D, Jucker M. The amyloid state of proteins in human diseases. Cell. 2012;148(6):1188-203.

2. Skinner M, Anderson J, Simms R, Falk R, Wang M, Libbey $C$, et al. Treatment of 100 patients with primary amyloidosis: a randomized trial of melphalan, prednisone, and colchicine versus colchicine only. Am J Med. 1996;100(3):290-8.

3. Falk RH, Comenzo RL, Skinner M. The systemic amyloidoses. N Engl J Med. 1997;337(13):898-909

4. Rapezzi C, Merlini G, Quarta CC, Riva L, Longhi S, Leone O, et al. Systemic cardiac amyloidoses: disease profiles and clinical courses of the 3 main types. Circulation. 2009;120(13):1203-12.

5. Gertz MA, Dispenzieri A, Sher T. Pathophysiology and treatment of cardiac amyloidosis. Nat Rev Cardiol. 2015;12(2):91-102.

6. Quarta CC, Solomon SD, Uraizee I, Kruger J, Longhi S, Ferlito M, et al. Left ventricular structure and function in transthyretin-related versus light-chain cardiac amyloidosis. Circulation. 2014;129(18):1840-9.

7. Fontana M, Pica S, Reant P, Abdel-Gadir A, Treibel TA, Banypersad SM, et al. Prognostic value of late gadolinium enhancement cardiovascular magnetic resonance in cardiac amyloidosis. Circulation. 2015;132(16):1570-9.

8. Brooks J, Kramer CM, Salerno M. Markedly increased volume of distribution of gadolinium in cardiac amyloidosis demonstrated by T1 mapping. J Magn Reson Imaging. 2013;38(6):1591-5.

9. Banypersad SM, Sado DM, Flett AS, Gibbs SD, Pinney JH, Maestrini V, et al. Quantification of myocardial extracellular volume fraction in systemic AL amyloidosis: an equilibrium contrast cardiovascular magnetic resonance study. Circ Cardiovasc Imaging. 2013;6(1):34-9.

10. Banypersad SM, Fontana M, Maestrini V, Sado DM, Captur G, Petrie A, et al. T1 mapping and survival in systemic light-chain amyloidosis. Eur Heart J. 2015;36(4):244-51.

11. Reese TG, Weisskoff RM, Smith RN, Rosen BR, Dinsmore RE, Wedeen VJ. Imaging myocardial fiber architecture in vivo with magnetic resonance. Magn Reson Med. 1995;34(6):786-91.

12. Hales PW, Schneider JE, Burton RA, Wright BJ, Bollensdorff C, Kohl P. Histo-anatomical structure of the living isolated rat heart in two contraction states assessed by diffusion tensor MRI. Prog Biophys Mol Biol. 2012;110(2-3):319-30.

13. Nielles-Vallespin S, Mekkaoui C, Gatehouse P, Reese TG, Keegan J, Ferreira PF, et al. In vivo diffusion tensor MRI of the human heart: reproducibility of breath-hold and navigator-based approaches. Magn Reson Med. 2013;70(2):454-65.

14. Wu MT, Tseng WY, Su MY, Liu CP, Chiou KR, Wedeen VJ, et al. Diffusion tensor magnetic resonance imaging mapping the fiber architecture remodeling in human myocardium after infarction: correlation with viability and wall motion. Circulation. 2006;114(10):1036-45.

15. McGill LA, Scott AD, Ferreira PF, Nielles-Vallespin S, Ismail T, Kilner PJ, et al. Heterogeneity of fractional anisotropy and mean diffusivity measurements by in vivo diffusion tensor imaging in Normal human hearts. PLoS One. 2015;10(7):e0132360

16. von Deuster C, Sammut E, Asner L, Nordsletten D, Lamata P. Stoeck CT, et al. Studying Dynamic Myofiber Aggregate Reorientation in Dilated 
Cardiomyopathy Using In Vivo Magnetic Resonance Diffusion Tensor Imaging. Circ Cardiovasc Imaging. 2016;9:10.

17. Nielles-Vallespin $S$, Khalique Z, Ferreira PF, de Silva R, Scott AD, Kilner $P$, et al. Assessment of myocardial microstructural dynamics by in vivo diffusion tensor cardiac magnetic resonance. J Am Coll Cardiol. 2017; 69(6):661-76.

18. Mekkaoui C, Jackowski MP, Kostis WJ, Stoeck CT, Thiagalingam A, Reese TG, et al. Myocardial Scar Delineation Using Diffusion Tensor Magnetic Resonance Tractography. J Am Heart Assoc. 2018;7(3).

19. Brenner DA, Jain M, Pimentel DR, Wang B, Connors LH, Skinner $M$, et al. Human amyloidogenic light chains directly impair cardiomyocyte function through an increase in cellular oxidant stress. Circ Res. 2004;94(8):1008-10.

20. Kramer CM, Barkhausen J, Flamm SD, Kim RJ, Nagel E. Standardized cardiovascular magnetic resonance (CMR) protocols 2013 update. J Cardiovasc Magn Reson. 2013;15(1):91.

21. Welsh CL, DiBella EV, Hsu EW. Higher-order motion-compensation for in vivo cardiac diffusion tensor imaging in rats. IEEE Trans Med Imaging. 2015; 34(9):1843-53.

22. Stoeck $C$, von Deuster $C$, Genet M, Atkinson D, Kozerke S. Second-order motion-compensated spin echo diffusion tensor imaging of the human heart. Magn Reson Med. 2016;75(4):1669-76.

23. Feinberg DA, Hoenninger JC, Crooks LE, Kaufman L, Watts JC, Arakawa M. Inner volume MR imaging: technical concepts and their application. Radiology. 1985;156(3):743-7.

24. von Deuster $C$, Stoeck CT, Genet M, Atkinson D, Kozerke S. Spin echo versus stimulated echo diffusion tensor imaging of the in vivo human heart. Magn Reson Med. 2015.

25. Moulin K, Croisille P, Feiweier T, Delattre BM, Wei H, Robert B, et al. In vivo free-breathing DTI and IVIM of the whole human heart using a real-time slice-followed SE-EPI navigator-based sequence: a reproducibility study in healthy volunteers. Magn Reson Med. 2016;76(1):70-82.

26. Messroghli DR, Radjenovic A, Kozerke S, Higgins DM, Sivananthan MU, Ridgway JP. Modified look-locker inversion recovery (MOLLI) for highresolution T1 mapping of the heart. Magn Reson Med. 2004;52(1):141-6.

27. Vishnevskiy V, Gass T, Szekely G, Tanner C, Goksel O. Isotropic Total variation regularization of displacements in parametric image registration. IEEE Trans Med Imaging. 2016.

28. Ferreira PF, Kilner PJ, McGill LA, Nielles-Vallespin S, Scott AD, Ho SY, et al. In vivo cardiovascular magnetic resonance diffusion tensor imaging shows evidence of abnormal myocardial laminar orientations and mobility in hypertrophic cardiomyopathy. J Cardiovasc Magn Reson. 2014;16:87.

29. Messroghli DR, Moon JC, Ferreira VM, Grosse-Wortmann L, He T, Kellman P, et al. Clinical recommendations for cardiovascular magnetic resonance mapping of $\mathrm{T} 1, \mathrm{~T}_{2}, \mathrm{~T}^{*}$ and extracellular volume: a consensus statement by the Society for Cardiovascular Magnetic Resonance (SCMR) endorsed by the European Association for Cardiovascular Imaging (EACVI). J Cardiovasc Magn Reson. 2017;19(1):75.

30. Martinez-Naharro A, Kotecha T, Norrington K, Boldrini M, Rezk T, Quarta C, et al. Native T1 and extracellular volume in transthyretin amyloidosis. JACC Cardiovasc Imaging. 2018.

31. Lin L, Li X, Feng J. Shen K-n, tian Z, sun J, et al. the prognostic value of T1 mapping and late gadolinium enhancement cardiovascular magnetic resonance imaging in patients with light chain amyloidosis. J Cardiovasc Magn Reson. 2018;20:2.

32. Basser PJ. Inferring microstructural features and the physiological state of tissues from diffusion-weighted images. NMR Biomed. 1995;8(7-8):333-44.

33. Nguyen C, Fan Z, Xie Y, Dawkins J, Tseliou E, Bi X, et al. In vivo contrast free chronic myocardial infarction characterization using diffusion-weighted cardiovascular magnetic resonance. J Cardiovasc Magn Reson. 2014;16:68.

34. Nguyen C, Liu M, Fan Z, Bi X, Kellman P, Li D, et al., editors. Vivo DiffusionWeighted MRI: Contrast-Free Detection of Myocardial Fibrosis in Hypertrophic Cardiomyopathy Patients Proc Intl Soc Mag Reson Med, vol. 23. Toronto. p. 2015.

35. Schelbert EB, Testa SM, Meier CG, Ceyrolles WJ, Levenson JE, Blair AJ, et al. Myocardial extravascular extracellular volume fraction measurement by gadolinium cardiovascular magnetic resonance in humans: slow infusion versus bolus. J Cardiovasc Magn Reson. 2011;13:16

36. Miller CA, Naish JH, Bishop P, Coutts G, Clark D, Zhao S, et al. Comprehensive validation of cardiovascular magnetic resonance techniques for the assessment of myocardial extracellular volume. Circ Cardiovasc Imaging. 2013;6(3):373-83.
37. Pagourelias ED, Mirea O, Vovas G, Duchenne J, Michalski B, Van Cleemput J, et al. Relation of regional myocardial structure and function in hypertrophic cardiomyopathy and amyloidois: a combined two-dimensional speckle tracking and cardiovascular magnetic resonance analysis. Eur Heart J Cardiovasc Imaging. 2018.

38. Gillmore JD, Maurer MS, Falk RH, Merlini G, Damy T, Dispenzieri A, et al. Nonbiopsy diagnosis of cardiac transthyretin amyloidosis. Circulation. 2016; 133(24):2404-12.

39. Kircher M, Ihne S, Brumberg J, Morbach C, Knop S, Kortum KM, et al. Detection of cardiac amyloidosis with (18) F-Florbetaben-PET/CT in comparison to echocardiography, cardiac MRI and DPD-scintigraphy. Eur J Nucl Med Mol Imaging. 2019

40. Stoeck CT, Kalinowska A, von Deuster C, Harmer J, Chan RW, Niemann M, et al. Dual-phase cardiac diffusion tensor imaging with strain correction. PLoS One. 2014;9(9):e107159.

\section{Publisher's Note}

Springer Nature remains neutral with regard to jurisdictional claims in published maps and institutional affiliations.

Ready to submit your research? Choose BMC and benefit from:

- fast, convenient online submission

- thorough peer review by experienced researchers in your field

- rapid publication on acceptance

- support for research data, including large and complex data types

- gold Open Access which fosters wider collaboration and increased citations

- maximum visibility for your research: over $100 \mathrm{M}$ website views per year

At BMC, research is always in progress.

Learn more biomedcentral.com/submissions 